\title{
Effects of electron cyclotron resonance etching on the ambient (100) GaAs surface
}

\author{
O. J. Glembocki and J. A. Tuchman \\ Naval Research Laboratory, Washington, DC 20375 \\ K. K. Ko and S. W. Pang \\ The University of Michigan, Ann Arbor, Michigan 48109 \\ A. Giordana and R. Kaplan \\ Naval Research Laboratory, Washington, DC 20375 \\ C. E. Stutz \\ Wright Patterson Laboratories, Dayton, Ohio 45433
}

(Received 16 January 1995; accepted for publication 8 March 1995)

\begin{abstract}
Photoreflectance has been used to study the electronic behavior of the ambient (100) GaAs surface and its modification by etching in a $\mathrm{Cl}_{2} / \mathrm{Ar}$ plasma generated by an electron-cyclotron resonance (ECR) source. We observed two pinning positions for ambient (100) GaAs, with $n$-GaAs pinning near midgap and $p$-GaAs pinning near the valance band. ECR etching shifts the Fermi level of $p$-GaAs toward midgap, but has little effect on $n$-GaAs. The surface modification is most influenced by the rf power. Auger electron spectroscopy indicates that the etching increases As at the GaAs/ oxide interface. We suggest that the Ga/As ratio controls the position of the Fermi level. (C) 1995 American Institute of Physics.
\end{abstract}

The decreased dimensions and increased complexity of semiconductor device structures have produced a need for better control over materials growth and processing. While fine control in epitaxial growth has existed for many years, this is not the case in the etching of semiconductors. In dry etching, there are two requirements for the production of electronic devices: large throughput and high aspect ratio geometries. These are fulfilled through a balance between the chemical and the physical sputtering components of dry etching processes. The chemical component consists of a reactive gas, which in combination with an ion beam significantly enhances the etch rate. Anisotropy is maintained by the sputtering component through energetic and directional ions. As a result, dry etching techniques such as reactive ion etching (RIE), chemically assisted ion beam etching (CAIBE), and plasma etching with an electron cyclotron resonance (ECR) source have replaced wet chemical etching in many areas of device fabrication.

A side effect of using energetic ions is that significant electronic damage can occur at the surface and in the bulk. In order to minimize etch damage, low energy ions (below 100 $\mathrm{eV}$ ) are required. At these levels, however, the etch rates in techniques such as CAIBE and RIE are significantly reduced. With an ECR source, on the other hand, ion fluxes and ion energies can be controlled independently. Therefore, it becomes possible to maintain high etch rates using low energy ions. Consequently, etch damage can be significantly reduced without sacrificing throughput.

In this work, we have studied changes in the electronic properties of GaAs surfaces induced by etching with a $\mathrm{Cl}_{2}$ /Ar plasma generated by an ECR source. The contactless optical spectroscopy of photoreflectance (PR) and specially designed GaAs multilayer structures ${ }^{1}$ were used to probe the Fermi-level pinning position at the GaAs/oxide interface, while Auger electron spectroscopy was used to identify the chemical composition of the interface.
The structures used in this study were undoped GaAs layers that were grown by molecular beam epitaxy (MBE) on $n^{+}$or $p^{+}$buffer layers and will be referred to as UN $\left(n^{+}\right.$ buffer) or UP ( $p^{+}$buffer). The buffer layers had doping densities in the range of $1-2 \times 10^{18} \mathrm{~cm}^{-3}$ and were $1.0 \mu \mathrm{m}$ thick. The undoped layers had a thickness of $1500 \AA$ for both the UN and UP structures. Because in the buffer the Fermi level is either in the valence (UP) or conduction (UN) bands while at the surface it is pinned near midgap, a constant electric field is produced in the undoped layer. This configuration ensures that changes in the surface potential are transferred directly to the electric field in the undoped region. The combination of a constant field and low lifetime broadening leads to photoreflectance spectra that are characterized by many Franz-Keldysh oscillations (FKO), allowing us to accurately determine the built-in field. ${ }^{1}$ Similar types of structures were recently used in studies of GaAs surfaces ${ }^{1,2}$ and their modification by sputtering, ${ }^{3,4}$ chemically assisted ion beam etching, ${ }^{5}$ and chemical passivation. ${ }^{6}$

The photoreflectance apparatus used in this study has been extensively described in the literature. ${ }^{7}$ The probe light was a monochromatic beam created by passing white light from a $100 \mathrm{~W}$ quartz halogen lamp through a SPEX $\frac{1}{4} \mathrm{~m}$ monochromator. The PR pump beam used to modulate the surface field was a $0.25 \mathrm{~mW}$ green $(5430 \AA) \mathrm{HeNe}$ laser mechanically chopped at $400 \mathrm{~Hz}$. The probe light was reflected from the sample and then detected with a Si photodiode. The signal from the photodiode was analyzed by a lock-in amplifier in order to extract the change in the reflectivity induced by the pump beam. The pump laser and probe beam had power densities of $50 \times 10^{-6} \mathrm{~W} / \mathrm{cm}^{2}$ and 1 $\times 10^{-6} \mathrm{~W} / \mathrm{cm}^{2}$, respectively.

Two sets of UN and UP samples were used in the experiments: one set was etched, while the other was not. The etched and unetched pairs were $1 \mathrm{~cm}^{2}$ neighbors from the same region of a larger wafer. PR measurements from all of 
the samples prior to etching showed variations in surface electric fields of less than 5\% across a wafer. This indicated that surface fields were uniform over the wafer.

Two samples, one UN and one UP, were always etched side by side in the ECR chamber. PR spectra were taken before and after the etching process. Auger electron spectroscopy was performed on a control piece and its etched neighbor.

The etching was performed in a plasma generated by an ECR source on top of an rf-powered electrode. The source is a multipolar plasma disk source surrounded by twelve permanent magnets, and is driven at $2.45 \mathrm{GHz}$. The ion energy can be independently controlled by the $13.56 \mathrm{MHz}$ rf power supply. The distance between the sample stage and the ECR source is adjustable between 7 and $27 \mathrm{~cm} . \mathrm{Cl}_{2}$ gas was introduced into the etch chamber through a gas ring situated 1 $\mathrm{cm}$ below the wafer stage, while Ar gas was introduced radially through the base of the ECR source.

The ion flux was varied by changing the microwave power, while maintaining a fixed self-induced dc bias, $\mid V_{\mathrm{dc}}$, of $100 \mathrm{~V}$. $\mathrm{A} \mathrm{Cl}_{2} / \mathrm{Ar}$ chemistry of $20 \% \mathrm{Cl}_{2}$ at $0.5 \mathrm{mTorr}$ and a $12 \mathrm{~cm}$ source-to-sample distance were used in the etching. In order to maintain a constant $\left|V_{\mathrm{dc}}\right|$, the rf power was increased from 31 to $47 \mathrm{~W}$ as the microwave power was increased from 0 to $500 \mathrm{~W}$. In all cases, the etch depth was kept constant at $\sim 350 \AA$. The ion energy was varied by changing the rf power. The sample-to-source distance was 15 $\mathrm{cm}$ and the $\mathrm{Cl}_{2} / \mathrm{Ar}$ mixture contained $30 \% \mathrm{Cl}_{2}$ at a pressure of $0.6 \mathrm{mTorr}$ and a microwave power of $50 \mathrm{~W}$. Increases in rf power from $20-300 \mathrm{~W}$ produced changes in $\left|V_{\mathrm{dc}}\right|$ from $77-$ $484 \mathrm{~V}$.

Photoreflectance can be used to measure the built-in electric field in a semiconductor because under a constant applied electric field the PR line shape can be approximated as $^{8}$

$\frac{\Delta R}{R} \sim V_{p} \cos \left[\frac{4}{3}\left(\frac{E-E_{g}}{\hbar \theta}\right)^{3 / 2}+\phi\right] \exp \left(-\frac{\Gamma\left(E-E_{g}\right)^{1 / 2}}{(\hbar \theta)^{3 / 2}}\right)$

where $E$ is the photon energy, $E_{g}$ is the energy gap, $\Gamma$ is the lifetime broadening, and $V_{p}$ is the photovoltage induced by the pump laser. The quantity $h \theta$ is the electro-optic energy, and is related to the electric field, $\mathbf{F}$, and the reduced interband effective mass along the direction of the electric field, $\mu_{\|}\left[=\left(\mu_{e}^{-1}+\mu_{h}^{-1}\right)^{-1}\right], \quad$ through $\hbar \theta=\left(e^{2}|F|^{2} \hbar^{2} / 2 \mu_{\|}\right)^{1 / 3}$. Because $\Delta R / R$ exhibits a periodic behavior, the built-in electric field can be determined from the period of the oscillations (FKO), using:

$$
n \pi=\frac{4}{3}\left(\frac{E_{n}-E_{g}}{\hbar \theta}\right)^{3 / 2}+\varphi
$$

where $E_{n}$ is the energy of the $n$th maxima.

It has been shown that in UN/UP structures, the photovoltage produced by the pump and probe beams can significantly reduce the barrier height. ${ }^{3,4}$ For GaAs this can be minimized by performing PR measurements at sample temperatures above $350 \mathrm{~K}^{2-4}$ In that case, the electric field obtained from Eq. (2) is related to the Fermi-level position by $V_{F}=F d-V_{d}$, where $d$ is the undoped layer thickness and

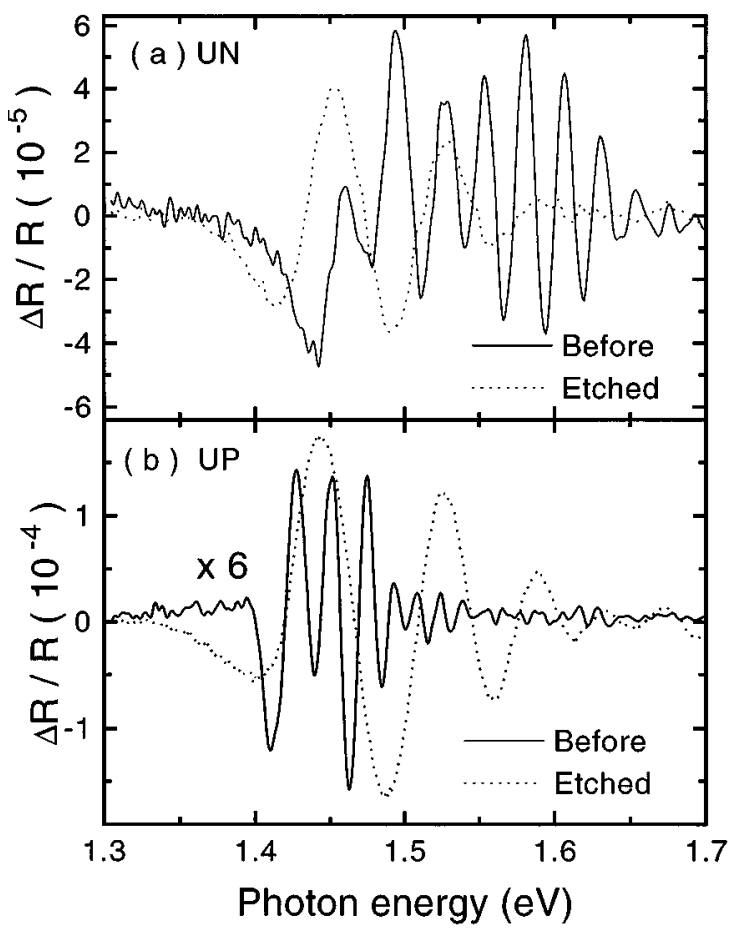

FIG. 1. Photoreflectance spectra for (a) UN and (b) UP structures before (solid) and after (dashed) ECR etching. The undoped layer thickness is 1500 $\AA$ for the unetched and $818 \AA$ for the etched samples.

$V_{d}$ is a correction due to band filling. For all of our PR measurements, we used sample temperatures of 300 and 400 $\mathrm{K}$.

Shown in Fig. 1 are $300 \mathrm{~K}$ photoreflectance spectra for unetched (solid) and etched (dashed) UN [Fig. 1(a)] and UP [Fig. 1(b)] structures. The etch conditions consisted of a microwave power of $50 \mathrm{~W}$ and an rf power of $300 \mathrm{~W}$. In each spectrum we see many FKO, indicating the existence of a sizable electric field in the undoped layer. The first thing to note about the data is that for the unetched samples, the period of the FKO for the UN structure is greater than for the UP sample, indicating different surface fields for $n$ - and $p$-GaAs, further suggesting different pinning positions for electrons and holes.

ECR etching increases the period of the FKO, indicating a larger surface field. This increase in field can be caused by two effects: a reduction in the thickness of the undoped region and an increase in the surface potential. It is important to note that in the case of the etched samples, the periods of the UN and UP structures are very similar, indicating that the pinning position of one of the samples has changed.

Shown in Fig. 2 are room temperature Fermi-level positions for the UN and UP samples as a function of the selfinduced bias, $\left|V_{\mathrm{dc}}\right|$ (rf power). The Fermi-level positions of the unetched samples agree well with previously reported data for (100) $\mathrm{GaAs}^{2,6}$ and show the two different pinning positions for $n$ - and $p$-GaAs. We also see that the Fermi-level position of the UN samples is hardly affected by changes in $\left|V_{\mathrm{dc}}\right|$ (ion energies), whereas the Fermi-level position of the UP sample moves away from the valence band and stabilizes near midgap. 


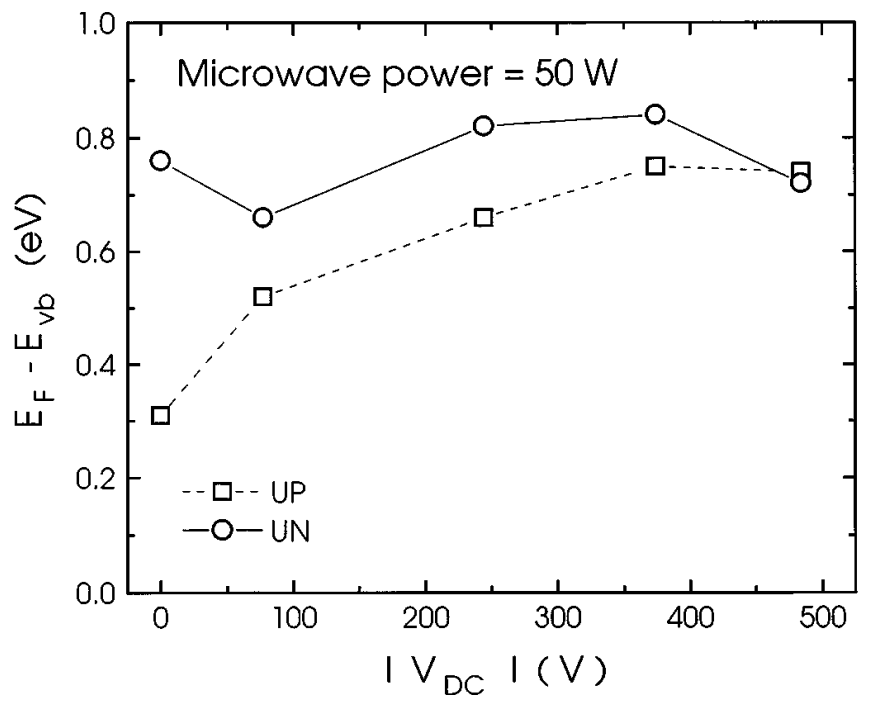

FIG. 2. The Fermi-level pinning position of the UN (circles) and the UP (squares) as a function of self-induced dc bias. All energies are relative to the top of the valence band $(\mathrm{vb})$.

We also studied the Fermi-level pinning position as a function of ion flux (microwave power). These samples were etched with a fixed $\left|V_{\mathrm{dc}}\right|$ of $100 \mathrm{~V}$. In this case, we found that the pinning is independent of the ion flux, suggesting that ion energy and not ion flux is responsible for changes in the electronic properties of the ECR etched surface.

Auger electron spectroscopy was performed on an unetched control sample and one that was etched with a selfinduced dc bias of $\left|V_{\mathrm{dc}}\right|=375 \mathrm{~V}$. Measurements were performed through the native oxide, as well as after Ar ion sputtering of the native oxide. Prior to sputtering, the intensity of oxygen Auger peaks was the same in the etched and control samples, indicating similar amounts of oxygen in both. We found $\mathrm{Ga} / \mathrm{As}$ ratios of 1.3 and 1.0 for the control and etched samples, respectively, suggesting that the stoichiometry of the oxides was different. After sputter removal of the oxide, the etched and control samples had $\mathrm{Ga} / \mathrm{As}$ ratios of 1.61 and 1.56 , respectively, which is characteristic of sputtered, bulk GaAs. This shows that the etched sample had an As rich oxide and allows us to correlate the motion of the Fermi level toward midgap to the presence of As at the GaAs/oxide interface.

These results have important implications. The first is the verification of the existence of two distinct states at the GaAs/oxide interface, which pin the Fermi level differently for $n$ - and $p$-GaAs. Pollak also observed two pinning sites at untreated (100) GaAs and their values of pinning positions are similar to those reported here. ${ }^{2}$ This is in contrast to earlier work that found that the Fermi level always pinned near midgap for both $n$ - and $p$-GaAs. The contrasting results can be understood if one assumes that the Fermi-level pinning position is dependent on the stoichiometry of the GaAs/ oxide interface. By ECR etching of GaAs, we have demonstrated that this indeed is true. The important result lies in the fact that ECR etching produces an oxide in which the Fermi level of the UP samples shifts toward midgap for $p$-type samples, and remains virtually unchanged for $n$-type materi- als. Furthermore, this shift is correlated with the presence of excess As at the GaAs/oxide interface. This allows us to suggest a model for the GaAs/oxide interface.

Certain chemical species, such as oxygen and chlorine, that might be expected to influence the Fermi level pinning can be eliminated by considering the Auger and PR results together. Because Auger spectroscopy shows no chlorine in the oxide, we believe that $\mathrm{Cl}$ induced interface states at the GaAs/oxide are highly unlikely. In the case of oxygen, even though adsorption of oxygen on (110) GaAs leads to different pinning positions in $n$ - and $p$-GaAs, ${ }^{8}$ Auger spectroscopy indicated similar levels of oxygen for both the etched and control samples.

We suggest that the surface states in ambient GaAs are not related to the presence of extrinsic chemical species, but are related to the stoichiometry of the GaAs/oxide interface. This is consistent with two different models for the pinning of semiconductors: the effective work function model (EWFM) of Freeouf and Woodall ${ }^{9}$ and the advanced unified defect model (UDM) of Spicer et al. ${ }^{10}$ In the EWFM, the pinning is determined by an effective work function obtained from the various species at the interface, while in the UDM the pinning is caused by antisite defects such as $\mathrm{As}_{\mathrm{Ga}}$ and $\mathrm{Ga}_{\mathrm{As}}$. In both cases, excess As pins the Fermi level near midgap, while excess Ga pins it near the valence band. While our measurements cannot distinguish between the two, one might speculate that the EWFM is more appropriate. This would be the case because the UDM would require the excess As or Ga to occupy very specific sites, whereas the EWFM does not.

In summary, we have used photoreflectance and Auger electron spectroscopies to characterize the etch damage in GaAs etched with an ECR source. We found that the etch had little effect on the Fermi level in $n$-GaAs, but caused a significant shift of the $p$-GaAs Fermi level toward midgap. In addition, we found that the oxides of the ECR-etched samples were As rich in comparison to native oxides on unetched samples. We suggest that the damage is related to changes in the stoichiometry of the oxide. Finally, it was shown that rf power most influences the surface damage, with little noticeable effects from the microwave power.

${ }^{1}$ C. Van Hoof, K. Deneffe, J. De Boeck, D. J. Arnet, and G. Borghs, Appl. Phys. Lett. 54, 608 (1989).

${ }^{2}$ F. H. Pollak, J. Vac. Sci. Technol. B 11, 1710 (1993); see also J. M. Woodall, Proc. Mater. Res. Soc. 324, 141 (1994).

${ }^{3}$ X. Yin, H. M. Chen, F. H. Pollak, Y. Cao, P. A. Montano, P. D. Kirchner, G. D. Pettit, and J. M. Woodall, J. Vac. Sci. Technol. B 9, 2114 (1991).

${ }^{4}$ X. Yin, H. M. Chen, F. H. Pollak, Y. Cao, P. A. Montano, P. D. Kirchner, G. D. Pettit, and J. M. Woodall, J. Vac. Sci. Technol. B 10, 131 (1991).

${ }^{5}$ O. J. Glembocki, J. A. Dagata, E. A. Dobisz and D. S. Katzer, Proc. Mater. Res. Soc. 236, 217 (1992) and O. J. Glembocki, J. A. Dagata, A. Giordana, D. S. Katzer, and C. E. Stutz, presented at the 1992 meeting of the American Vacuum Society, Chicago, IL, December, 1992.

${ }^{6}$ O. J. Glembocki, Proc. Soc. Photo-Opt. Instrument. Eng. 1286, 1 (1990).

${ }^{7}$ D. E. Aspnes, Phys. Rev. B 153, 972 (1967). and R. N. Bhattacharya, H. Shen, P. Parayanthal, F. H. Pollak, T. Coutts, and H. Aharoni, Phys. Rev. B 37, 4044 (1988). 5, 1119 (1987).

${ }^{8}$ K. Stiles, D. Mao, and A. Kahn, J. Vac. Sci. Technol. B 6, 1170 (1988).

${ }^{9}$ J. L. Freehouf and J. M. Woodall, Appl. Phys. Lett. 39, 727 (1981).

${ }^{10}$ W. E. Spicer, N. Newman, C. J. Spindt, Z. Liliental-Webber, and E. R. Weber, J. Vac. Sci. Technol. B 8, 2084 (1990). 